\title{
CCD OBSERVATIONS AND ANALYSIS OF THE W UMA TYPE BINARY SS ARIETIS
}

\author{
WONYONG HAN \\ Korea Astronomy Observatory \\ CHUN-HWEY KIM AND JAE WOO LEE \\ Department of Astronomy \& Space Science, Chungbuk National University \\ AND \\ HO-IL KIM AND WOO-BAIK LEE \\ Korea Astronomy Observatory
}

The BVR CCD observations of W UMa-type eclipsing binary SS Arietis were made for ten nights from November 1996 to December 1996 at the Sobaeksan Astronomy Observatory. From the observed light curves, nine new times of minimum lights were derived from the Kwee and van Woerden's method. Improved light elements for this system were determined from these minimum lights with all the published minima. The analysis of the times of minima of SS Ari confirms that the orbital period of SS Ari has been suffering from a sinusoidal variation as the suggestions of other previous investigators (Kaluzny \& Pojmanski 1984, Demircan \& Selam 1993). The calculation shows that the cyclic period change has a period of about $56.3 \mathrm{yrs}$ with an amplitude of about $0 .{ }^{d} 052$. The period variation has been discussed in terms of two potential mechanisms : 1) the light-time effect due to a hypothetical third body and 2) deformations in the convective envelope of a magnetically active component. The $B V R$ light curves of SS Ari observed for about one month showed the existence of cycle to cycle light variations. Long-term light changes of SS Ari are discussed in terms of the period variation of the binary system. 